\title{
Correction to: Age-Appropriate Wisdom?
}

\section{Ethnobiological Knowledge Ontogeny in Pastoralist Mexican Choyeros}

\author{
Eric Schniter ${ }^{1}$ (D) . Shane J. Macfarlan 2,3,4 . Juan J. Garcia ${ }^{5}$. \\ Gorgonio Ruiz-Campos ${ }^{6}$. Diego Guevara Beltran ${ }^{7}$. Brenda B. Bowen ${ }^{4,8}$. \\ Jory C. Lerback ${ }^{8}$
}

Published online: 13 May 2021

(c) Springer Science+Business Media, LLC, part of Springer Nature 2021

\section{Correction to: Human Nature https://doi.org/10.1007/s12110-021-09387-8}

In the article published as https://doi.org/10.1007/s12110-021-09387-8 the captions and images for Figures 1 and 2 were reversed. The following shows the correct placement of the two images and captions.

\section{Study Sample and Ecology}

Our study was conducted across four ranching communities (Santa Maria de Toris, San Pedro de la Presa, La Higuera, and La Soledad) in the southern Sierra de La Giganta mountain range within the municipality of La Paz, Baja California Sur, México (Fig. 1).

The online version of the original article can be found at https://doi.org/10.1007/s12110-021-09387-8

$\triangle$ Eric Schniter

eschniter@gmail.com

1 Economic Science Institute, Chapman University, Orange, CA, USA

2 Department of Anthropology, University of Utah, Salt Lake City, UT, USA

3 Center for Latin American Studies, University of Utah, Salt Lake City, UT, USA

4 Global Change and Sustainability Center, University of Utah, Salt Lake City, UT, USA

5 Department of Education, Culture, and Society, University of Utah, Salt Lake City, UT, USA

6 Departamento de Biología, Universidad Autónoma de Baja California, Ensenada, Baja California, México

7 Department of Psychology, University of Arizona, Tucson, AZ, USA

8 Department of Geology and Geophysics, University of Utah, Salt Lake City, UT, USA 


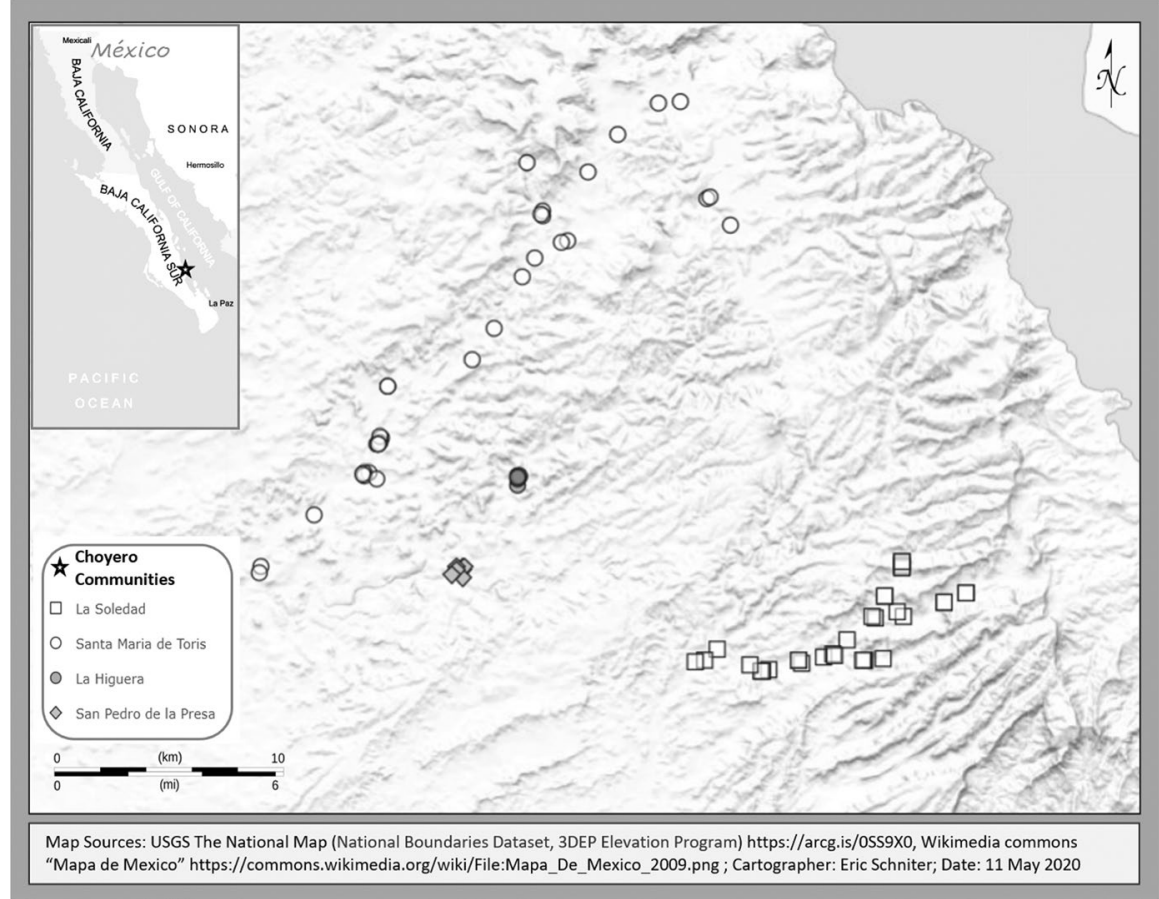

Fig. 1 Choyero ranches and communities in the southern Sierra de La Giganta of Baja California Sur, México

\section{Choyero Demographics and Fertility}

Consistent with these estimates for parent ages at peak offspring dependency, our sampling at the household level from across 70 ranches indicates that the number of coresident dependent offspring ( 2 on average) relying on a head of household is greatest for heads of household in the 35- to 45-year-old age range (Fig. 2). The number of dependent offspring observed for heads of households of a given age is always less than the number of expected offspring because a large proportion of our sample included adults with no coresident dependents. 


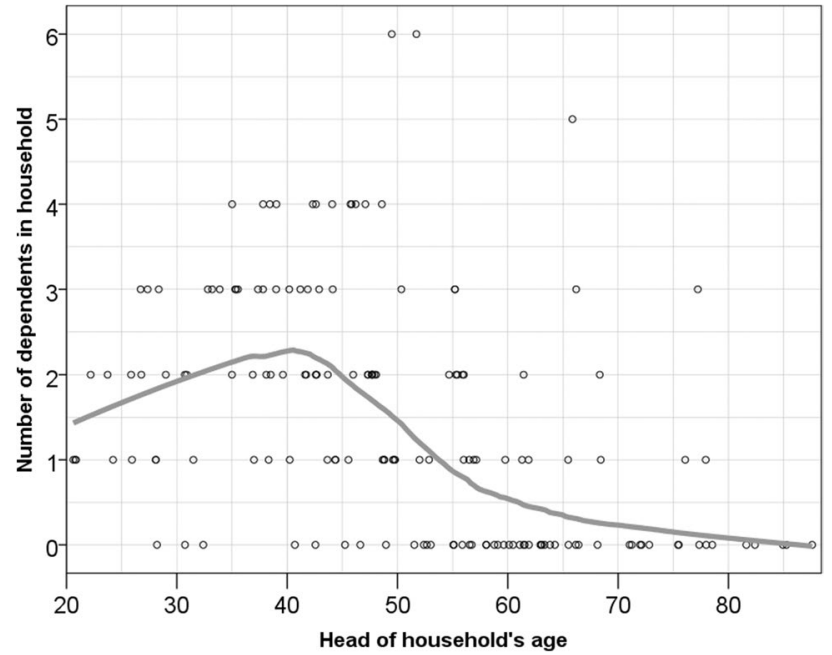

Fig. 2 Loess smooth lines (50\% fit) showing number of dependent offspring for each Choyero head of household $(n=143)$

The original article has been corrected.

Publisher's Note Springer Nature remains neutral with regard to jurisdictional claims in published maps and institutional affiliations. 